\title{
Superior Mesenteric Artery Occlusion by a Thrombus That Was Successfully Treated Using Interventional Radiology: A Case Report
}

\author{
Kazumasa Emori ${ }^{\mathrm{a}}$, Nobuhiro Takeuchi ${ }^{\mathrm{a}, \mathrm{e}}$, Makoto Yoshitani ${ }^{\mathrm{a}}$, Junichi Soneda ${ }^{\mathrm{a}}$, \\ Kaori Mohrib ${ }^{\text {, Yusuke Nomura }}{ }^{\mathrm{c}}$, Masanori Takada ${ }^{\mathrm{d}}$
}

\begin{abstract}
A thrombus in the superior mesenteric artery (SMA) is a critical condition that requires immediate diagnosis and treatment. We present a case of a thrombus in SMA that was successfully treated by interventional radiology. A 63-year-old male with a history of myocardial infarction, diabetes mellitus, hypertension, and gastric ulcers was admitted to our hospital complaining of severe upper abdominal pain. He had smoked 20 cigarettes per day for 40 years. Upon arrival, his blood pressure was extremely high (254/120 mm Hg). Noncontrast computed tomography (CT) revealed no apparent cause for his symptom. Electrocardiography revealed Q waves in V1 and V2 without ST elevation in other leads. Myocardial angina could not be ruled out. Immediately after the administration of heparin sodium, nicardipine, and nitrates, his symptoms alleviated. On day 2, upper endoscopy revealed multiple gastric ulcers, and a proton pump inhibitor was administered. On day 3, severe upper abdominal pain recurred. Contrast-enhanced CT revealed a thrombus in the proximal SMA. Prostaglandin E1 was intravenously administered. On day 4, abdominal angiography was performed, revealing an occluded thrombus in the proximal SMA with developed circulation in peripheral intestinal arteries. The thrombus was successfully resolved by arterial administration of urokinase and a balloon catheter. On day 5, postoperative CT revealed dilated small bowels. Subsequently, an ileus tube was inserted. The overall clinical course was uneventful, and oral feeding along with oral administration of aspirin and warfarin was initiated on day 18 .
\end{abstract}

Keywords: Superior mesenteric artery; Occlusion; Thrombus; Interventional radiology

Manuscript accepted for publication February 02, 2017

aDepartment of Internal Medicine, Kobe Tokushukai Hospital, Tarumi, Kobe, Japan

bDepartment of Laboratory Medicine, Kobe Tokushukai Hospital, Tarumi, Kobe, Japan

${ }^{\mathrm{c}}$ Department of Internal Medicine, Kawasaki Hospital, Kobe, Japan

${ }^{\mathrm{d} D e p a r t m e n t}$ of Cardiology, Kawasaki Hospital, Kobe, Japan

${ }^{e}$ Corresponding Author: Nobuhiro Takeuchi, Department of Internal Medicine, Kobe Tokushukai Hospital, 1-3-10 Kamitakamaru, Tarumi-Ku, Kobeshi, Hyogo 655-0017, Japan. Email: nobuhiro.takeuchi@tokushukai.jp

doi: https://doi.org/10.14740/jmc2746w

\section{Introduction}

Acute mesenteric artery occlusion is a critical condition with a high mortality rate of $60-80 \%$ which requires urgent diagnosis and treatment [1]. Acute superior mesenteric artery (SMA) occlusion, if left untreated, may lead to irreversible intestinal ischemia and subsequently, result in intestinal necrosis. Therefore, surgical intervention is sometimes required. Recently, with the development of interventional radiology (IVR), thrombus aspiration using a catheter is usually attempted unless the intestinal necrosis is progressive. In SMA occlusion, if a thrombus or emboli is located in the proximal SMA without a developing peripheral circulation, intestinal ischemia leads to intestinal necrosis followed by immediate death. Alternatively, if collateral arteries are established after a thrombus in SMA, progression of intestinal ischemia may be alleviated. Here we discuss a case of SMA occlusion by a thrombus that was successfully treated using IVR.

\section{Case Report}

A 63-year-old man arrived at our department by ambulance complaining of upper abdominal pain. His past medical records included myocardial infarction, type 2 diabetes mellitus, hypertension, and gastric ulcers.

On admission, his blood pressure was 254/120 mm Hg, heart rate was 86 beats/min with regular rhythm, body temperature was $36.8{ }^{\circ} \mathrm{C}$, and oxygen saturation was $97 \%$ under $2 \mathrm{~L} / \mathrm{min}$ oxygen inhalation. The initial clinical examination revealed a weight of $60.5 \mathrm{~kg}$ and a height of $166 \mathrm{~cm}$. Inspection of the palpebral conjunctiva showed no evidence of anemia. Auscultation of the heart revealed no murmurs. Auscultation of the lungs revealed no rales or other abnormalities. Physical examination of his abdomen revealed tenderness over the upper abdomen without any signs of peritonitis.

Blood chemistry analyses revealed severely elevated white blood cell counts $(18,060$ cells $/ \mu \mathrm{L})$, dense red blood (red blood cell count, $605 \times 10^{4}$ cells $/ \mu \mathrm{L}$ and hemoglobin, $19.9 \mathrm{~g} /$ dL), mildly elevated lactate dehydrogenase (312 IU/L), severely elevated glucose levels $(256 \mathrm{mg} / \mathrm{dL})$, and mildly elevated fibrin and fibrinogen degradation product $(7 \mu \mathrm{g} / \mathrm{mL})$ and D-dimer levels $(2.1 \mu \mathrm{g} / \mathrm{mL})$. 

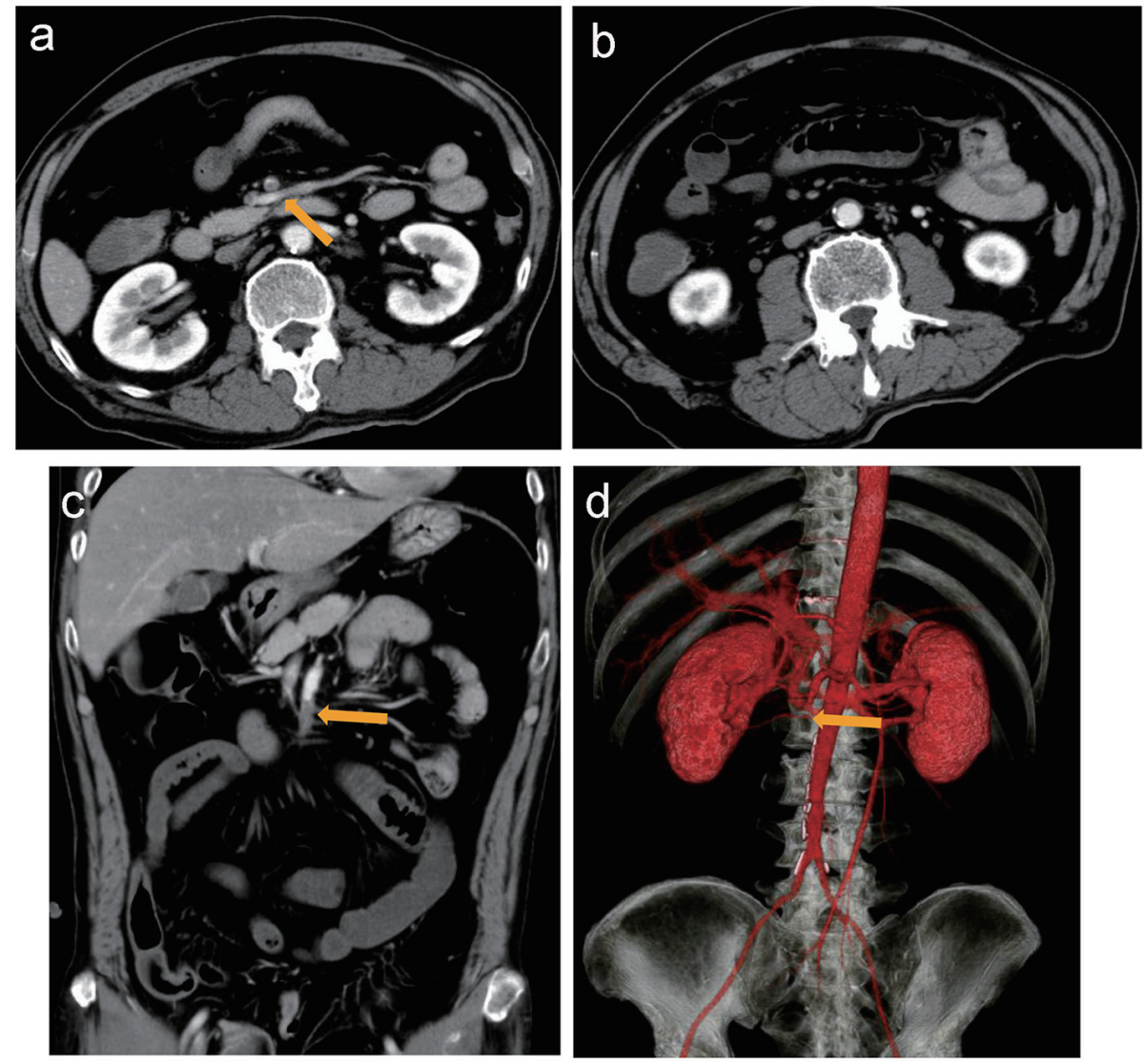

Figure 1. Contrast-enhanced computed tomography (CT) reveals a suspected thrombus (arrow) in the main trunk of the superior mesenteric artery (a) and dilated small intestines (b). Coronal section (c) and three-dimensional contrast-enhanced CT (d) suggest that the thrombus is located in the main trunk of SMA at the level of the second branch of the jejunal artery (arrow).

Electrocardiography revealed a normal sinus rhythm with Q waves in V1 and V2, without ST elevation in other leads. Myocardial angina could not be excluded. After the administration of nicardipine and nitrates, his symptom improved. On day 2 , upper endoscopy revealed multiple gastric ulcers, therefore, a proton pump inhibitor was administered and fasting treatment was resumed. On day 3 , he presented with recurrent severe upper abdominal pain. Contrast-enhanced computed tomography (CT) was performed, revealing a suspected thrombus in the main trunk of SMA (Fig. 1a) as well as extensively dilated small bowels (Fig. 1b). Coronal sections and three-dimensional contrast-enhanced CT indicated that the thrombus was located in the second branch of the jejunal artery (Fig. 1c, d). Immediately, prostaglandin E1 and heparin sodium were intravenously administered. On day 4, dilated small bowels were apparent on CT (Fig. 2a). Doppler US showed dilated and edematous small intestines with decreased blood flow (Fig. 2b), raising suspicion of intestinal ischemia. His abdominal pain continued. On
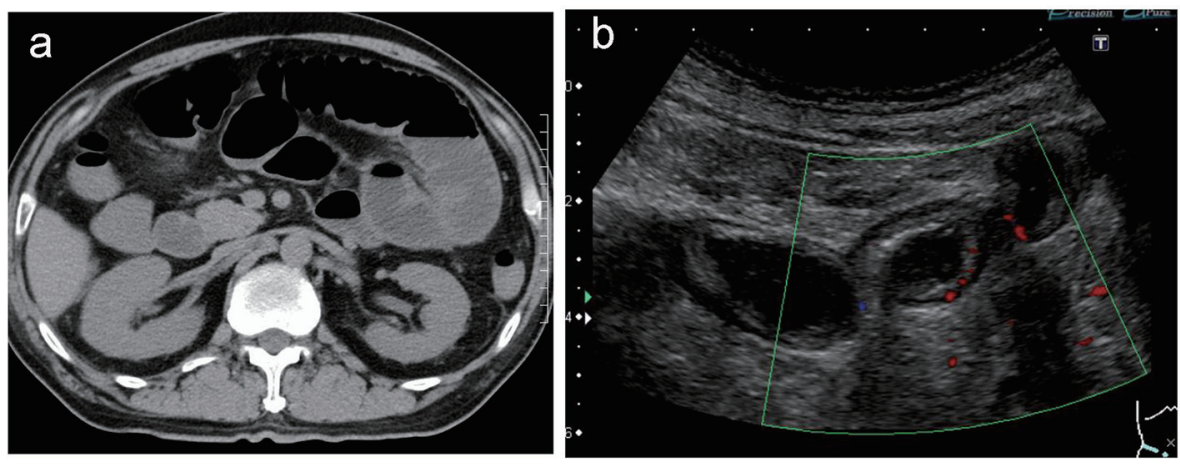

Figure 2. Non-contrast computed tomography reveals extensively dilated small intestines (a). Doppler ultrasonography reveals edematous and dilated small intestines with reduced intestinal perfusion (b). 

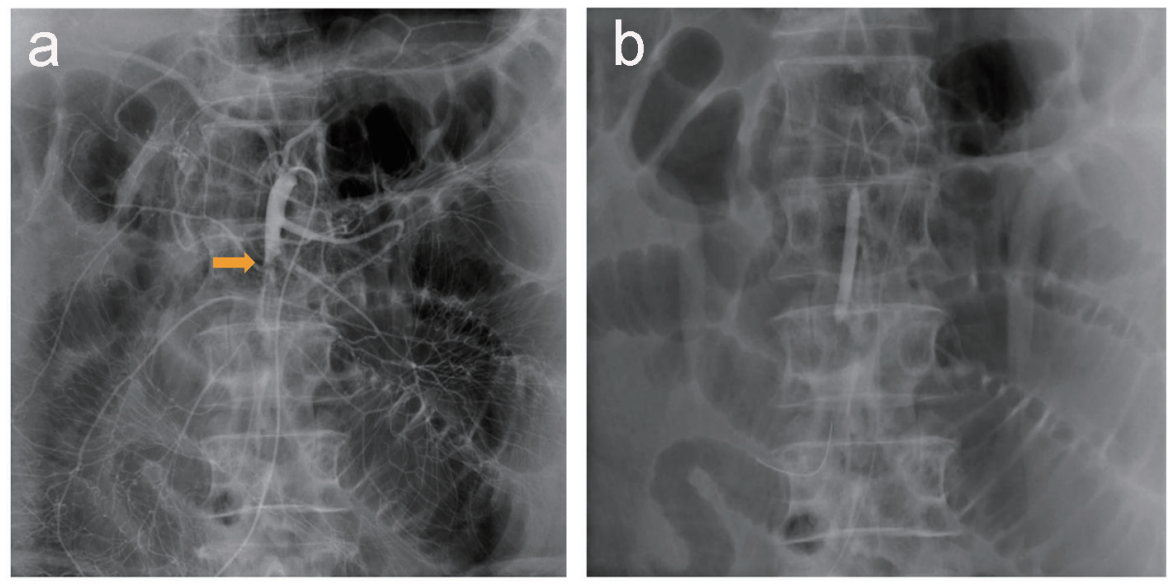

Figure 3. Abdominal angiography reveals a thrombus in the main trunk of SMA at the level of the second branch of the jejunal artery, with preserved peripheral circulation because of developed collateral arteries (a). A 0.019-inch guide wire and a balloon catheter are successfully delivered to the lesion (b).

the same day, abdominal angiography was performed, revealing a thrombus in the main trunk of SMA at the level of the second branch of the jejunal artery as well as preserved peripheral circulation due to developed collateral arteries (Fig. 3a). Subsequently, IVR was performed. A 0.019-inch guide wire was passed into the lesion with ease and a peripheral balloon catheter was delivered. After repeated dilatation of the balloon, urokinase (480,000 units) was administered from the catheter. On day 5, X-ray revealed dilated small bowels (Fig. 4a); therefore, an ileus tube was inserted to deflate the bowel pressure (Fig. 4b). On day 6, angiography and ultrasonography revealed that the thrombus in SMA had totally disappeared, but irregular stenotic artery lesions remained (Fig. 5a, b).

Administration of antibiotics and fasting treatment were continued after thrombolysis. Although the peak C-reactive protein (CRP) levels were severely elevated $(47.3 \mathrm{mg} / \mathrm{dL})$, there was no sign of peritoneal irritation or evidence of intestinal necrosis. CRP levels gradually dropped and clinical symptoms improved. On day 18, oral intake of anti-coagulant and antiplatelet agents was initiated to prevent reformation of thromboses. The subsequent clinical course was uneventful.

\section{Discussion}

SMA occlusion is an acute abdomen disease with a high mortality rate of $60-80 \%$ [1]. Acute SMA occlusion is caused by the occlusion of the mesenteric artery which can lead to extensive intestinal necrosis and panperitonitis, leading to septic shock and disseminated intravascular coagulation.

Accurate diagnosis rates of SMA occlusion by angiography are much higher than those by contrast-enhanced CT. Angiography is efficient not only for diagnosis but also for the subsequent treatment, including angioplasty or thrombolysis. The application of IVR in SMA occlusion is limited to intestinal ischemia without intestinal necrosis; if SMA occlusion is accompanied by intestinal necrosis, surgical intervention, including vascular revascularization and intestinal resection,
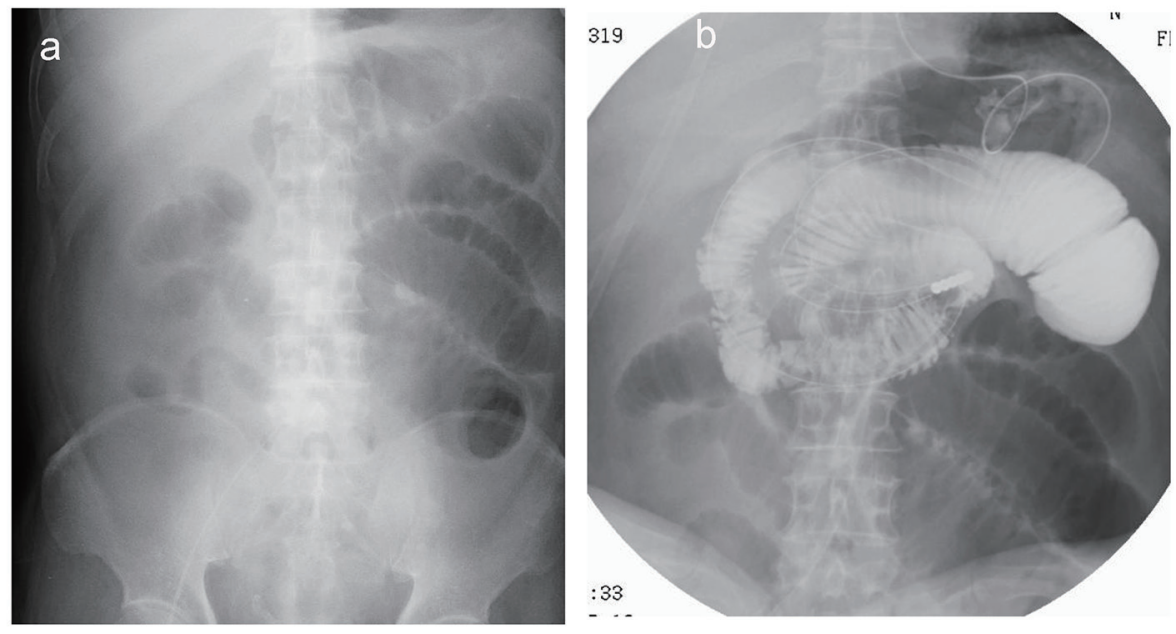

Figure 4. X-ray reveals dilated small bowels (a) that require placement of an ileus tube to deflate the bowel pressure (b). 

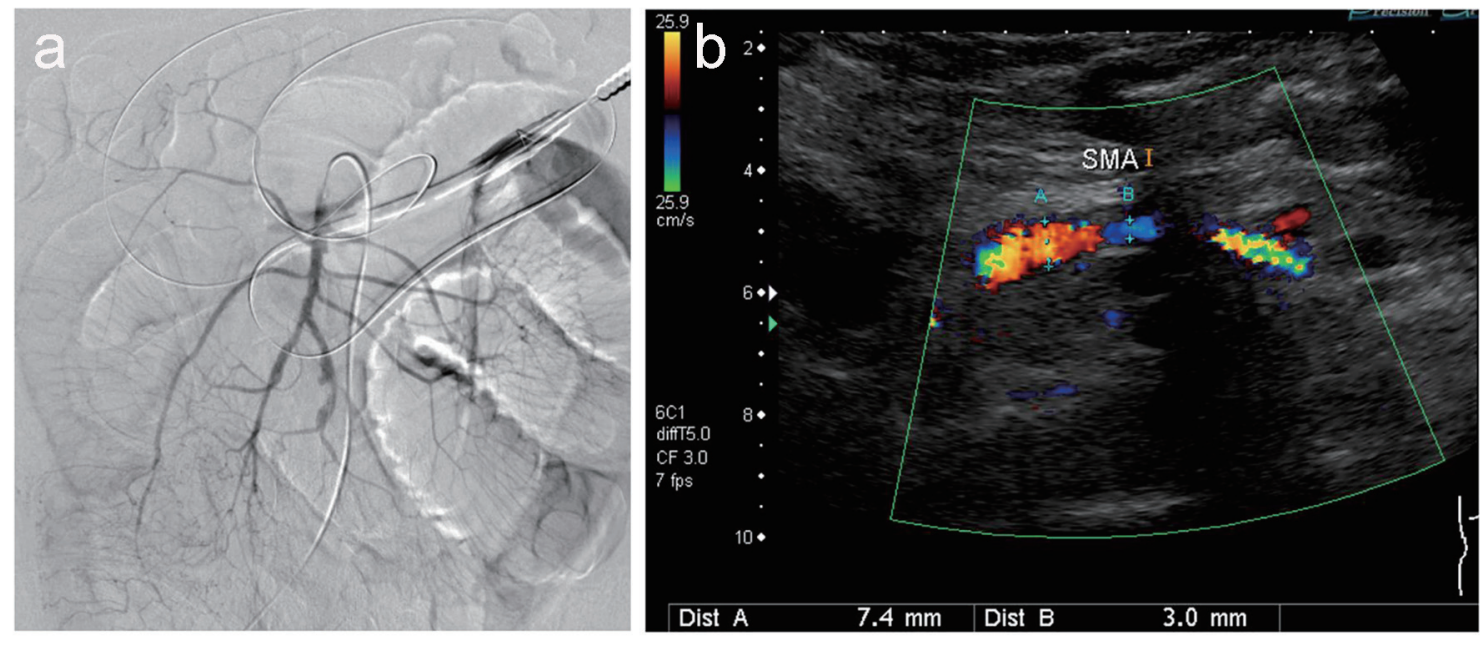

Figure 5. Angiography (a) and ultrasonography (b) revealed no thrombus, but irregular stenotic arteries are evident.

is required [2]. Evidence of intestinal necrosis is supported by clinical presentation (signs of peritoneal irritation or shock), biochemistry analysis (elevated white blood cell counts; increased aspartate aminotransferase, creatinine phosphokinase levels, and lactate dehydrogenase levels; or presence of metabolic acidosis), or radiological findings (pneumatosis intestinalis, intrahepatic portal venous gas, or intraperitoneal free air). In general, the golden time for treating a thrombus in SMA, where intestinal ischemia can be reversed and intestinal resection can be avoided, is deemed to be within $10-12 \mathrm{~h}$ after the onset [3]. However, it has become clear that this principle does not always hold true depending on the site(s), degree(s), or severity of SMA occlusion.

Acute SMA occlusion, unless treated within $10-12 \mathrm{~h}$ from the onset, is considered to cause irreversible intestinal necrosis. In our patient, thrombolytic therapy was initiated at $72 \mathrm{~h}$ after the clinical manifestation. Despite the main trunk of SMA being occluded by a thrombus, blood flow in the small intestines was preserved because of the development of collateral arteries. Acute SMA occlusion has been classified into the following three types, depending on the location of the occlusion [2]: type A, where the occlusion is in the origin of the main trunk of SMA or proximal to the middle or right colic artery; type $\mathrm{B}$, where the occlusion is between right colic artery and ileocolic arteries; and type $\mathrm{C}$, where the occlusion is distal to the ileocolic artery. Type A is not expected to establish collateral circulation, thereby developing intestinal necrosis within $5 \mathrm{~h}$ and requires a laparotomy rather than thrombolysis. In contrast, type $\mathrm{B}$ and type $\mathrm{C}$ yield favorable results of revascularization even if treatment is initiated after the golden time. It can be safely said that the indication for thrombolytic therapy can be broadened depending on the causes and locations of ischemia and extent of collateral vessels. In our case, this patient might have chronic stenotic locations at the main trunk of SMA that originated from atherosclerosis, and thromboembolism might occur in those regions.

Several problems may occur after thrombolysis with IVR: 1) reperfusion injury after recanalization [4], 2) chronic thromboses resistant to thrombotic agents, 3) lack of assessment regarding recovered intestinal circulation, and 4) reformation of thromboses after recanalization. Reperfusion injury after recanalization remains to be investigated and is currently under research. It is possible, whether SMA is occluded by fresh or chronic thromboses, to successfully resolve the lesion using a guide wire. Thus far, there are few optimal methods for estimating intestinal perfusion; however, a combination of laboratory analysis and imaging modalities, including contrast-enhanced CT and Doppler US, improves the assessment of the intestinal circulation. Anti-coagulant therapy after the recanalization is essential to prevent thromboses from reforming. If acute SMA occlusion is treated with thrombolysis, subsequent intravenous administration of heparin sodium is required. Angioplasty of acute SMA occlusion yields high success rates of recanalization (approximately $80 \%$ ). However, more than $50 \%$ cases of acute SMA occlusion treated with angioplasty lead to restenosis of the underlying lesions; therefore, stent placement after balloon dilatation is recommended [5-7].

\section{Conclusion}

We have presented a case of acute SMA occlusion by a thrombus that was successfully treated by IVR. Endovascular treatment may be possible even after $12 \mathrm{~h}$ have passed since the clinical onset, if collateral blood flow was established. However, immediate diagnosis and treatment is required to prevent further intestinal ischemia. It is also important to note that acute mesenteric artery occlusion is one of the differential diagnoses of upper abdominal pain.

\section{References}

1. Yasuhara H. Acute mesenteric ischemia: the challenge of gastroenterology. Surg Today. 2005;35(3):185-195.

2. Moteki K, Ishitobi K, Seki M, et al. Acute Superior Mesenteric Arterial Occlusion - Occlusion Sites and its Clinical Course. Journal of Abdominal Emergency Medicine. 
1996;16(2):427-432.

3. Batellier J, Kieny R. Superior mesenteric artery embolism: eighty-two cases. Ann Vasc Surg. 1990;4(2):112116.

4. Zimmerman BJ, Granger DN. Reperfusion injury. Surg Clin North Am. 1992;72(1):65-83.

5. Lindblad B, Lindh M, Chuter T, Ivancev K. Superior mesenteric artery occlusion treated with PTA and stent placement. Eur J Vasc Endovasc Surg. 1996;11(4):493-
495.

6. Maleux G, Wilms G, Stockx L, Vancleemput J, Baert AL. Percutaneous recanalization and stent placement in chronic proximal superior mesenteric artery occlusion. Eur Radiol. 1997;7(8):1228-1230.

7. Bocchini T, Hoffman J, Zuckerman D. Mesenteric ischemia due to an occluded superior mesenteric artery treated by percutaneous transluminal angioplasty. J Clin Gastroenterol. 1995;20(1):86-88. 